\title{
Description and properties of curve-based monotone functions
}

\author{
Mikel Sesma-Sara ${ }^{1,2}$, Laura De Miguel ${ }^{1,2}$, Antonio Francisco Roldán López de \\ Hierro $^{3}$, Jana Špirková ${ }^{4}$, Radko Mesiar ${ }^{5,6}$, and Humberto Bustince ${ }^{1,2}$ \\ 1 Public University of Navarra, Spain \\ 2 Institute of Smart Cities (UPNA), Spain \\ \{mikel.sesma, laura.demiguel, bustince\}@unavarra.es \\ 3 University of Granada, Spain \\ aroldan@ugr.es \\ 4 Matej Bel University, Slovakia \\ jana.spirkova@umb.sk \\ ${ }^{5}$ Slovak University of Technology in Bratislava, Slovakia \\ 6 University of Ostrava, Czech Republic \\ mesiar@math.sk
}

\begin{abstract}
Curve-based monotonicity is one of the lately introduced relaxations of monotonicity. As directional monotonicity regards monotonicity along fixed rays, which are given by real vectors, curve-based monotonicity studies the increase of functions with respect to a general curve $\alpha$. In this work we study some theoretical properties of this type of monotonicity and we relate this concept with previous relaxations of monotonicity.
\end{abstract}

Keywords: Curve-based monotonicity $\cdot$ weak monotonicity $\cdot$ directional monotonicity $\cdot$ aggregation function.

\section{Introduction}

Aggregation operators are functions that aim at finding a single value to represent a collection of $n$ numbers, and, since this is a desired feature in many processes, they have been largely studied and applied [6,8,14].

Aggregation functions need to be increasing with respect to every argument. However, according to various works in the literature $[5,19]$, that condition may be too restrictive as it restricts some functions, that are fit to fuse information, to enter the framework of aggregation functions. That is the reason why, there is a trend towards relaxing the monotonicity constraint in the definition of aggregation function $[7,13]$.

In that attempt, some generalizations of monotonicity have been proposed. For example, weak monotonicity [19] is a relaxed form of monotonicity, as it only asks for the value of a function to increase in the case where all the arguments have increased by the same amount. This notion can be seen as monotonicity defined by the vector $(1, \ldots, 1)$ and, considering any vector $\vec{r} \in \mathbb{R}^{n}$, instead, 
directional monotonicity was defined [5]. Directional monotonicity is the requirement that is demanded to functions in order to be considered pre-aggregation functions [11]. Lately, more different relaxations of monotonicity have appeared $[1,4,16,18]$, some of which have been used to construct edge detectors $[4,17]$ and fuzzy ruled-based classification systems $[10,12]$.

In this work, we discuss a generalization of directional monotonicity: curvebased monotonicity [9]. Rather than directions given by vectors, curve-based monotonicity studies the monotonicity of functions along general curves $\alpha$ : $[0,1] \rightarrow \mathbb{R}^{n}$. Clearly, lines are particular instances of curves and, hence, curvebased monotonicity generalizes directional monotonicity.

We study some theoretical properties of curve-based monotonicity, including the situation of functions that are monotone with respect to two curves $\alpha$ and $\beta$, which are also monotone with respect to the combination of such curves.

This paper is organized in the following manner: first, we present some preliminaries, including the definitions of various relaxations of monotonicity. In Section 3, we recall the concept of curve-based monotonicity. In Section 4 we show a collection of properties of curve-based monotone functions and, in Section 5 , we discuss the case of composition of two curves. Finally, we present some concluding remarks and future perspectives.

\section{Preliminaries}

Let $n \in \mathbb{N}$ such that $n>1$. On the one hand, we use $\mathbf{x}=\left(x_{1}, \ldots, x_{n}\right)$ to denote points in $[0,1]^{n}$ and we set $\mathbf{0}=(0, \ldots, 0)$ and $\mathbf{1}=(1, \ldots, 1) \in[0,1]^{n}$. On the other hand, we use $\vec{r} \in \mathbb{R}^{n}$ to refer to vectors that denote directions in the real space.

In this work, we consider curves on $\mathbb{R}^{n}$ as functions defined on a closed real interval, i.e., $\alpha:[0,1] \rightarrow \mathbb{R}^{n}$. Note that the choice of the domain, $[0,1]$, could have been any other closed real interval $[a, b] \subset \mathbb{R}$, as any curve $\alpha$ defined on $[0,1]$ can be re-parametrized to be defined on $[a, b]$. Additionally, we only consider curves $\alpha$ such that $\alpha(0)=\mathbf{0}$.

Given a curve $\alpha:[0,1] \rightarrow \mathbb{R}^{n}$, all the components $\alpha_{1}, \ldots, \alpha_{n}$ of $\alpha$ can be seen as curves on $\mathbb{R}: \alpha_{i}:[0,1] \rightarrow \mathbb{R}$ for all $1 \leq i \leq n$.

The concept of monotonicity is intimately related to the notion of order. We use the standard partial order in $\mathbb{R}^{n}$ (and, hence, in $[0,1]^{n}$ ), i.e., given $\mathbf{x}, \mathbf{y} \in$ $[0,1]^{n}$, we say that $\mathbf{x} \leq \mathbf{y}$ if $x_{i} \leq y_{i}$ for al $1 \leq i \leq n$. Thus, we can define the concept of standard monotonicity for a function $f:[0,1]^{n} \rightarrow[0,1]$.

Definition 1. A function $f:[0,1]^{n} \rightarrow[0,1]$ is said to be increasing (resp. decreasing) if for all $\mathbf{x}, \mathbf{y} \in[0,1]^{n}$ such that $\mathbf{x} \leq \mathbf{y}$ it holds that $f(\mathbf{x}) \leq f(\mathbf{y})$ (resp. $f(\mathbf{x}) \geq f(\mathbf{y})$ ).

Note that with the terms increasing and decreasing we do not refer to strict monotonicity.

As stated in the Introduction, we aim at relaxing the monotonicity condition of aggregation functions. An aggregation function is a function $A:[0,1]^{n} \rightarrow[0,1]$ 
such that $A(\mathbf{0})=0, A(\mathbf{1})=1$ and $A$ is increasing in the sense of Definition 1 . Let us now present some of relaxations of monotonicity.

Definition 2 ([19]). A function $f:[0,1]^{n} \rightarrow[0,1]$ is said to be weakly increasing (resp. weakly decreasing) if for all $\mathbf{x} \in[0,1]^{n}$ and $c>0$ such that $\mathbf{x}+c \mathbf{1} \in[0,1]^{n}$ it holds that $f(\mathbf{x}) \leq f(\mathbf{x}+c \mathbf{1})$ (resp. $f(\mathbf{x}) \geq f(\mathbf{x}+c \mathbf{1})$ ).

Note that, although robust estimators of location [15], which are used in statistics, are generally not monotone, they are shift-invariant and shift-invariance implies weak monotonicity.

Nevertheless, although monotonicity with respect to all arguments may be too restrictive for certain applications, our expectation with respect to the behaviour of means requires that some monotonicity-like condition is satisfied, e.g., in the case of robust estimators of location shift-invariance is required

Remark 1. If a function $f:[0,1]^{n} \rightarrow[0,1]$ is monotone, then it is also weakly monotone, i.e., standard increasingness (resp. decreasingness) implies weak increasingness (resp. decreasingness).

However, the converse does not hold. For example, the mode function, with the convention of taking the minimum if all the inputs are different, is not generally increasing. Indeed, $(0,0.2,0.3,0.3,0.3) \leq(0.2,0.2,0.3,0.4,0.5)$ but

$$
\operatorname{mode}(0,0.2,0.3,0.3,0.3)=0.3>0.2=\operatorname{mode}(0.2,0.2,0.3,0.4,0.5) .
$$

But, the mode function satisfies a certain kind of monotonicity, as its value increases whenever all the inputs increase by the same amount, i.e., the mode is a weakly increasing function.

Weak monotonicity can be seen as monotonicity along the ray $(1, \ldots, 1)$. When, we consider a general vector $\overrightarrow{0} \neq \vec{r} \in \mathbb{R}^{n}$ instead, we obtain the notion of directional monotonicity.

Definition 3 ([5]). Let $\overrightarrow{0} \neq \vec{r} \in \mathbb{R}^{n}$ and $f:[0,1]^{n} \rightarrow[0,1]$. We say that $f$ is $\vec{r}$-increasing (resp. $\vec{r}$-decreasing) if for all $\mathbf{x} \in[0,1]^{n}$ and $c>0$ such that $\mathbf{x}+c \vec{r} \in[0,1]^{n}$, it holds that $f(\mathbf{x}) \leq f(\mathbf{x}+c \vec{r})($ resp. $f(\mathbf{x}) \geq f(\mathbf{x}+c \vec{r}))$.

A function $f$ that is both $\vec{r}$-increasing and $\vec{r}$-decreasing for a certain $\overrightarrow{0} \neq$ $\vec{r} \in \mathbb{R}^{n}$ is said to be $\vec{r}$-constant.

A function that satisfies the boundary conditions of aggregation functions and is directionally increasing with respect to some direction $\overrightarrow{0} \neq r \in \mathbb{R}^{n}$ is said to be a pre-aggregation function [10-12].

It is also interesting to study the directions for which a function is directionally increasing. If the set of such directions forms a cone, we say that a function is cone increasing. This concept was originally defined for positive cones $C \subset\left(\mathbb{R}^{+}\right)^{n}$, but the generalization to any cone $C \subset \mathbb{R}^{n}$ is straight. Recall that a subset $C \subset \mathbb{R}^{n}$ is said to be a cone if for each $\mathbf{x} \in C$ it holds that $a \mathbf{x} \in C$ for all $a \geq 0$. 
Definition 4 ([1]). Let $\emptyset \neq C \subset \mathbb{R}^{n}$ be a cone. A function $f:[0,1]^{n} \rightarrow[0,1]$ is said to be cone increasing with respect to $C$ (resp. cone decreasing) if $f$ is $\vec{r}$-increasing (resp. $\vec{r}$-decreasing) for all vectors $\vec{r} \in C$.

Clearly, increasing functions in the standard sense are cone increasing with respect to the cone $\left(\mathbb{R}^{+}\right)^{n}$.

The interested reader can find numerous examples of functions that satisfy each of the monotonicity conditions in $[1-3,5,19]$.

\section{Curve-based monotonicity}

In the same manner that directional monotonicity is a generalization of weak monotonicity considering general directions $\vec{r}$ instead of $\overrightarrow{1}$, we can think of an even more general concept by considering curves in the space.

Definition 5 ([9]). Let $\alpha:[0,1] \rightarrow \mathbb{R}^{n}$ be a curve such that $\alpha(0)=\mathbf{0}$. A function $f:[0,1]^{n} \rightarrow[0,1]$ is said to be $\alpha$-increasing (resp. $\alpha$-decreasing) if $f(\mathbf{x}) \leq f(\mathbf{x}+\alpha(t))$ (resp. $f(\mathbf{x}) \geq f(\mathbf{x}+\alpha(t)))$ for all $\mathbf{x} \in[0,1]^{n}$ and all $0<t \leq 1$ such that $\mathbf{x}+\alpha(s) \in[0,1]^{n}$ for all $0<s \leq t$.

From this point forward, we assume that all curves $\alpha:[0,1] \rightarrow \mathbb{R}^{n}$ satisfy the condition $\alpha(0)=\mathbf{0}$, unless otherwise stated.

If a function $f$ is both $\alpha$-increasing and $\alpha$-decreasing for a given curve $\alpha$ : $[0,1] \rightarrow \mathbb{R}^{n}$, then $f$ is said to be $\alpha$-constant. For curves defined on an open interval, see [9].

Note that, by Definition 5 , for a function $f$ to be $\alpha$-increasing, once the curve leaves the unit hypercube $[0,1]^{n}$, it has no influence in the property of $\alpha$-monotonicity of $f$. Indeed, the condition that must hold is $f(\mathbf{x}) \leq f(\mathbf{x}+\alpha(t))$ provided that all the points $\mathbf{x}+\alpha(s) \in[0,1]^{n}$ for all $0<s \leq t$. Therefore, the points $\mathbf{x}+\alpha(t) \notin[0,1]^{n}$, even in the case that the curve eventually returns to take values within $[0,1]^{n}$, do not influence the condition of $\alpha$-monotonicity. This is shown in Figure 1.

Remark 2. Straight lines (or segments of straight lines) are a particular instance of curve. Hence, curve-based monotonicity is a generalization of directional monotonicity. Indeed, let $\overrightarrow{0} \neq \vec{r} \in \mathbb{R}^{n}$ and $f:[0,1]^{n} \rightarrow[0,1]$ be an $\vec{r}$-increasing function. Then, if we set $\alpha:[0,1] \rightarrow \mathbb{R}^{n}$ to be the curve given by $\alpha(t)=t \vec{r}$, we obtain that $f$ is $\alpha$-increasing. In particular, weak monotonicity is also a particular case of curve-based monotonicity.

Further, we derive the following result from the definitions of curve-based monotonicity and directional monotonicity.

Proposition 1. Let $\alpha:[0,1] \rightarrow \mathbb{R}^{n}$ be a curve such that, given $t_{0} \in[0,1]$, $\alpha(t)=\left(r_{1} t, \ldots, r_{n} t\right)$ for all $t \in\left[0, t_{0}\right]$, where $r_{1}, \ldots, r_{n} \in \mathbb{R}$, which defines $a$ direction $\vec{r}$. If a function $f:[0,1]^{n} \rightarrow[0,1]$ is $\alpha$-increasing, then $f$ is $\vec{r}_{-}$ increasing. 


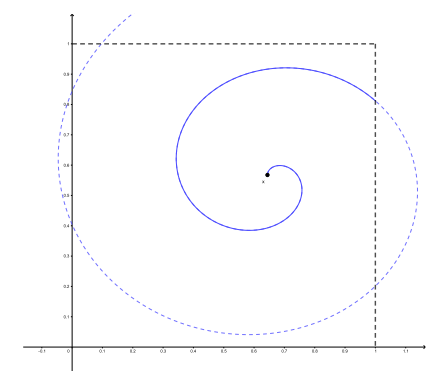

Fig. 1. Example of points $\mathbf{x}+\alpha(t)$ that have influence on the $\alpha$-monotonicity of functions (in solid blue) and points that do not (dashed blue).

Let us stress that the concept of curve-based montonicity, presented in Definition 5, differs from monotonicity along a curve $\alpha$. Namely, the fact that the value of a function $f$ increases along the graph of a certain curve $\alpha$ implies that the function $f$ is $\alpha$-increasing, but the converse does not hold in general as the next example shows.

Example 1. The arithmetic mean, defined in $[0,1]^{n}$ by

$$
A_{n}\left(x_{1}, \ldots, x_{n}\right)=\frac{1}{n} \sum_{i=1}^{n} x_{i},
$$

is an example of $\alpha$-monotone function for a curve $\alpha$ that satisfies certain properties.

Specifically, the arithmetic mean is $\alpha$-increasing for all curves $\alpha:[0,1] \rightarrow \mathbb{R}^{n}$ such that

$$
\sum_{i=1}^{n} \alpha_{i}(t) \geq 0
$$

for all $0 \leq t \leq 1$. Furthermore, the arithmetic mean is $\alpha$-decreasing for all curves $\alpha$ such that $\sum_{i=1}^{n} \alpha_{i}(t) \leq 0$, for all $0 \leq t \leq 1$. Consequently, $A$ is $\alpha$-constant for every curve $\alpha$ such that $\sum_{i=1}^{n} \alpha_{i}(t)=0$, for all $0 \leq t \leq 1$.

The arithmetic mean serves also to show that $\alpha$-monotonicity does not coincide with monotonicity along the graph of the curve $\alpha$. Let $\alpha:[0,1] \rightarrow \mathbb{R}^{2}$ be a curve given by

$$
\alpha(t)= \begin{cases}(t, 0), & \text { if } 0 \leq t \leq 0.5 \\ (0.5,0.5-t), & \text { if } 0.5<t \leq 1\end{cases}
$$

Clearly, since $\alpha$ verifies condition (1), the arithmetic mean $A_{2}$ is $\alpha$-increasing. However, the value of $A_{2}$ does not increase along the graph of $\alpha$. Indeed, consider $(0,0) \in[0,1]^{2}$ and let $g:[0,1] \rightarrow[0,1]$ be the function that represents the values 
of $A_{2}$ along the graph of $\alpha$. Thus, it is given by

$$
\begin{aligned}
g(t) & =A_{2}\left(0+\alpha_{1}(t), 0+\alpha_{2}(t)\right) \\
& = \begin{cases}\frac{t}{2}, & \text { if } 0 \leq t \leq 0.5, \\
\frac{1-t}{2}, & \text { if } 0.5<t \leq 1,\end{cases}
\end{aligned}
$$

and, clearly, $g$ is decreasing for $0.5<t \leq 1$.

\section{Properties of curve-based monotone functions}

In this section we study some relevant properties of curve-based monotone functions and we discuss how curve-based monotonicity relates to other relaxations of monotonicity.

Proposition 2. Let $f:[0,1]^{n} \rightarrow[0,1]$ be a function, $\alpha:[0,1] \rightarrow \mathbb{R}^{n}$ be a curve and $\varphi:[0,1] \rightarrow[0,1]$ be a strictly increasing one-to-one mapping. Then, $f$ is $\alpha$-increasing (resp. $\alpha$-decreasing) if and only if $f$ is $(\alpha \circ \varphi)$-increasing (resp. $(\alpha \circ \varphi)$-decreasing $)$.

The next result characterizes standard monotonicity in terms of curve-based monotonicity.

Proposition 3. Let $f:[0,1]^{n} \rightarrow[0,1]$. Then, $f$ is increasing (resp. decreasing) if and only if $f$ is $\alpha$-increasing (resp. $\alpha$-decreasing) for all curves $\alpha:[0,1] \rightarrow \mathbb{R}^{n}$ such that $\alpha(t) \geq \mathbf{0}$ for all $0 \leq t \leq 1$.

Proof. Let $f:[0,1]^{n} \rightarrow[0,1]$ be an increasing (resp. decreasing) function, $\alpha:$ $[0,1] \rightarrow \mathbb{R}^{n}$ be a curve such that $\alpha(t) \geq \mathbf{0}$ for all $1 \leq t \leq n$ and let $\mathbf{x} \in[0,1]^{n}$ and $t \in[0,1]$ such that $\mathbf{x}+\alpha(s) \in[0,1]^{n}$ for all $0<s \leq t$. Since $\alpha(t) \geq \mathbf{0}$, then $\mathbf{x} \leq \mathbf{x}+\alpha(t)$. Therefore, since $f$ is increasing (resp. decreasing), it holds that $f(\mathbf{x}) \leq f(\mathbf{x}+\alpha(t))($ resp. $f(\mathbf{x}) \geq f(\mathbf{x}+\alpha(t)))$.

For the converse, assume that $f$ is $\alpha$-increasing (resp. $\alpha$-decreasing) for every curve $\alpha:[0,1] \rightarrow \mathbb{R}^{n}$ such that $\alpha(t) \geq \mathbf{0}$ for all $1 \leq t \leq n$. Now, let $\mathbf{x}, \mathbf{y} \in[0,1]^{n}$ such that $\mathbf{x} \leq \mathbf{y}$. We can set a curve $\alpha:[0,1] \rightarrow \mathbb{R}^{n}$ given by $\alpha(t)=t \mathbf{y}-t \mathbf{x}$ for all $0 \leq t \leq 1$. Thus,

$$
\begin{gathered}
f(\mathbf{x}) \leq f(\mathbf{x}+\alpha(t))=f((1-t) \mathbf{x}+t \mathbf{y}) \\
(\text { resp. } f(\mathbf{x}) \geq f(\mathbf{x}+\alpha(t))=f((1-t) \mathbf{x}+t \mathbf{y})) .
\end{gathered}
$$

In particular, for $t=1$, we conclude that $f(\mathbf{x}) \leq f(\mathbf{y})($ resp. $f(\mathbf{x}) \geq f(\mathbf{y}))$ and therefore $f$ is increasing (resp. decreasing).

As a consequence, in the case where all the components of the curve $\alpha$ are identical, we recover the notion of weak monotonicity. 
Corollary 1. Let $f:[0,1]^{n} \rightarrow[0,1]$. Then, $f$ is weakly increasing (resp. weakly decreasing) if and only if $f$ is $\alpha$-increasing (resp. $\alpha$-decreasing) for all curves $\alpha:[0,1] \rightarrow \mathbb{R}^{n}$ such that $\alpha_{i}(t)=\alpha_{j}(t) \geq \mathbf{0}$ for all $i, j \in\{1, \ldots, n\}$ and all $0 \leq t \leq 1$.

The following is an example of a function that is $\alpha$-increasing for a curve $\alpha$ : $[0,1] \rightarrow \mathbb{R}^{2}$ but fails to be directionally monotone with respect to any direction $\vec{r} \in \mathbb{R}^{2}$.

Example 2. Let $f:[0,1]^{2} \rightarrow[0,1]$ given by

$$
f(x, y)=\left\{\begin{array}{l}
1, \text { if } x=y=0.25 \\
0, \text { otherwise }
\end{array}\right.
$$

and let $\alpha:[0,1] \rightarrow \mathbb{R}^{2}$ be the curve given by

$$
\alpha(t)= \begin{cases}(0.3,0), & \text { if } 0<t \leq 0.3 \\ (t, 0), & \text { otherwise }\end{cases}
$$

The function $f$ is not $\vec{r}$-increasing for any direction $\vec{r} \in \mathbb{R}^{2}$ since it has a strict global maximum at the point $(0.25,0.25)$ and the value of $f$ goes from 1 to 0 from the point $(0.25,0.25)$ in any direction.

However, $f$ is $\alpha$-increasing as, clearly, there does not exist any point $(0.25,0.25) \neq$ $(x, y) \in \mathbb{R}^{2}$ such that $(x, y)+\alpha(t)=(0.25,0.25)$ for all $0 \leq t \leq 1$.

Let us now present two examples of $\alpha$-increasing functions for certain curves $\alpha:[0,1] \rightarrow \mathbb{R}^{n}$.

Example 3. (1) Let $f:[0,1]^{2} \rightarrow[0,1]$ be the function given by

$$
f(x, y)=\frac{1+3 x-y^{2}}{4}
$$

for all $x, y \in[0,1]$.

It is not hard to check that this function is $\alpha$-increasing for any curve $\alpha$ : $[0,1] \rightarrow \mathbb{R}^{2}$ such that $\alpha_{1}:[0,1] \rightarrow \mathbb{R}$ is increasing and $\alpha_{2}:[0,1] \rightarrow \mathbb{R}$ is decreasing; or $\alpha_{2}$ is increasing and $\alpha_{1}(t) \geq \frac{2 \alpha_{2}(t)+\alpha_{2}^{2}(t)}{3}$ for all $0 \leq t \leq 1$; or the graph of $\alpha$ is located the fourth quadrant of the plane.

(2) Let $f:[0,1]^{2} \rightarrow[0,1]$ be given by

$$
f(x, y)=\frac{x}{y+1},
$$

for all $x, y \in[0,1]$. This function is $\alpha$-increasing for any curve $\alpha:[0,1] \rightarrow \mathbb{R}^{2}$ whose graph is located on the fourth quadrant of the plane.

The following result characterizes the notion of $\alpha$-monotonicity for a function $f:[0,1]^{n} \rightarrow[0,1]$ in terms of the values that it takes in the proximity of each point of the domain. Specifically, it shows an upper and a lower bound for each point. 
Theorem 1. Let $f:[0,1]^{n} \rightarrow[0,1]$ and $\alpha:[0,1] \rightarrow \mathbb{R}^{n}$ be a curve that is continuous at $t=0$. Then, $f$ is $\alpha$-increasing if and only if for all $\mathbf{x} \in[0,1]^{n}$ and all $r, s \in[0,1]$ such that

i) $\mathbf{x}-\alpha(r)+\alpha(t) \in[0,1]^{n}$, for all $0 \leq t \leq r$, and

ii) $\mathbf{x}+\alpha(t) \in[0,1]^{n}$, for all $0 \leq t \leq s$;

it holds that

$$
f(\mathbf{x}-\alpha(r)) \leq f(\mathbf{x}) \leq f(\mathbf{x}+\alpha(s)) .
$$

Proof. Given a curve $\alpha:[0,1] \rightarrow \mathbb{R}^{n}$, it is clear that if a function $f:[0,1]^{n} \rightarrow$ $[0,1]$ verifies (3) for all $\mathbf{x} \in[0,1]^{n}$ and all $r, s \in[0,1]$ that satisfy $i$ ) and $\left.i i\right)$, then $f$ is also $\alpha$-increasing.

For the converse, let us suppose that $f$ is $\alpha$-increasing and let $r, s \in[0,1]$ such that $i$ ) and $i i$ ) hold. From $i i$ ) and the fact that $f$ is $\alpha$-increasing, it is clear that $f(\mathbf{x}) \leq f(\mathbf{x}+\alpha(s))$. Similarly, from $i)$, since $\mathbf{x}-\alpha(r)+\alpha(t) \in[0,1]^{n}$, for all $0 \leq t \leq r$, in particular, for $t=0$, it holds that $\mathbf{x}-\alpha(r) \in[0,1]^{n}$. Thus, since $f$ is $\alpha$-increasing, we obtain that

$$
f(\mathbf{x}-\alpha(r)+\alpha(r)) \geq f(\mathbf{x}-\alpha(r)) .
$$

Hence, $f(\mathbf{x}-\alpha(r)) \leq f(\mathbf{x})$ and this completes the proof for the two inequalities in (3).

\section{Curve-based monotonicity with respect to the composition of curves}

In this section, we study the conditions of curve-based monotonicity of functions with respect to the composition of two, or more, curves. By composition of two curves, we refer to the curve whose graph goes through the first curve and, then, through the second (see Figure 2), i.e., given two curves $\alpha, \beta:[0,1] \rightarrow \mathbb{R}^{n}$, we define their composition $\alpha \beta:[0,1] \rightarrow \mathbb{R}^{n}$ by

$$
\alpha \beta(t)= \begin{cases}\alpha(2 t), & \text { if } 0 \leq t \leq 0.5, \\ \alpha(1)+\beta(2 t-1), & \text { if } 0.5<t \leq 1 .\end{cases}
$$

The next result shows that if a function is curve-based monotone with respect to two different curves, then it is also curve-based monotone with respect to the combination of the two curves.

Theorem 2. Let $\alpha:[0,1] \rightarrow \mathbb{R}^{n}$ and $\beta:[0,1] \rightarrow \mathbb{R}^{n}$ be two curves and let $f:[0,1]^{n} \rightarrow[0,1]$ be a function. If $f$ is $\alpha$-increasing (resp. $\alpha$-decreasing) and $\beta$-increasing (resp. $\beta$-decreasing), then $f$ is $\alpha \beta$-increasing (resp. $\alpha \beta$-decreasing).

Proof. Let $\alpha, \beta:[0,1] \rightarrow \mathbb{R}^{2}$ be two curves and let $f:[0,1]^{n} \rightarrow[0,1]$ be $\alpha$ - and $\beta$-increasing. Let $\mathbf{x} \in[0,1]^{n}$ and $t \in[0,1]$ such that $\mathbf{x}+\alpha \beta(s) \in[0,1]^{n}$ for all $0 \leq s \leq t$. If $t \leq 0.5$, by $\alpha$-increasingness of $f$ it holds that

$$
f(\mathbf{x}+\alpha \beta(t))=f(\mathbf{x}+\alpha(2 t)) \geq f(\mathbf{x}) .
$$




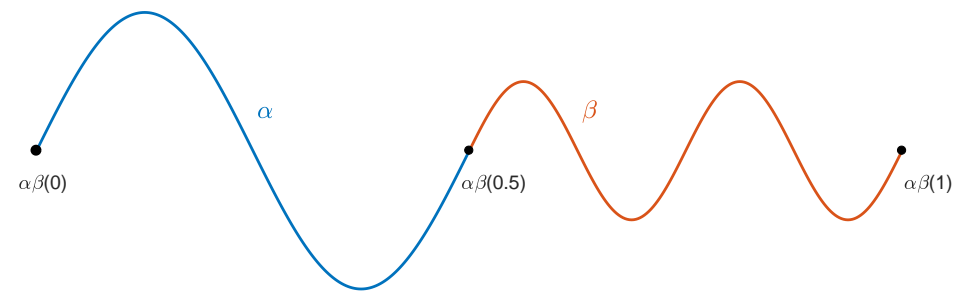

Fig. 2. Graph of a composition curve $\alpha \beta$, constructed from two curves $\alpha$ and $\beta$ as in (4).

On the other hand, if $t>0.5$, it holds that

$$
f(\mathbf{x}+\alpha \beta(t))=f(\mathbf{x}+\alpha(1)+\beta(2 t-1)),
$$

and, since $\mathbf{x}+\alpha(1) \in[0,1]^{n}$ and $\mathbf{x}+\alpha(1)+\beta(2 t-1) \in[0,1]^{n}$, by the $\beta$ increasingness of $f$ we derive that

$$
f(\mathbf{x}+\alpha(1)+\beta(2 t-1)) \geq f(\mathbf{x}+\alpha(1)) .
$$

Therefore, from (5), (6) and the fact that $f$ is $\alpha$-increasing we derive that $f(\mathbf{x}+$ $\alpha \beta(t)) \geq f(\mathbf{x})$.

\section{Conclusion}

We have discussed the concept of curve-based monotonicity, which is an extension of a recently introduced relaxation of monotonicity: directional monotonicity. We have presented some remarks and clarifications regarding the notion of $\alpha$-monotonicity, for a certain curve $\alpha$, as well as some theoretic properties. We have also shown some examples of functions that satisfy curve-based monotonicity for specific curves.

With respect to perspectives for future research, our aim is to investigate how this concept could benefit some applied problems. In particular, we intend to use curve-based monotone functions in the are of computer vision.

\section{Acknowledgements}

This work is supported by the research group FQM268 of Junta de Andalucía, by the project TIN2016-77356-P (AEI/FEDER, UE), by the Slovak Scientific Grant Agency VEGA no. 1/0093/17 Identification of risk factors and their impact on products of the insurance and savings schemes, by Slovak grant APVV-14-0013, and by Czech Project LQ1602 "IT4Innovations excellence in science". 


\section{References}

1. Beliakov, G., Calvo, T., Wilkin, T.: Three types of monotonicity of averaging functions. Knowledge-Based Systems 72, 114-122 (2014). https://doi.org/10.1016/j.knosys.2014.08.028

2. Beliakov, G., Spirková, J.: Weak monotonicity of Lehmer and Gini means. Fuzzy Sets and Systems 299, 26-40 (2016). https://doi.org/10.1016/j.fss.2015.11.006

3. Beliakov, G., Calvo, T., Wilkin, T.: On the weak monotonicity of Gini means and other mixture functions. Information Sciences 300, 70-84 (2015)

4. Bustince, H., Barrenechea, E., Sesma-Sara, M., Lafuente, J., Dimuro, G.P., Mesiar, R., Kolesárová, A.: Ordered directionally monotone functions. Justification and application. IEEE Transactions on Fuzzy Systems 26(4), 2237-2250 (2018). https://doi.org/10.1109/TFUZZ.2017.2769486

5. Bustince, H., Fernandez, J., Kolesárová, A., Mesiar, R.: Directional monotonicity of fusion functions. European Journal of Operational Research 244(1), 300-308 (2015). https://doi.org/10.1016/j.ejor.2015.01.018

6. Elkano, M., Sanz, J.A., Galar, M., Pekala, B., Bentkowska, U., Bustince, H.: Composition of interval-valued fuzzy relations using aggregation functions. Information Sciences 369, 690-703 (2016)

7. Gagolewski, M.: Data fusion: theory, methods, and applications. Institute of Computer Science Polish Academy of Sciences (2015)

8. García-Lapresta, J., Martínez-Panero, M.: Positional voting rules generated by aggregation functions and the role of duplication. International Journal of Intelligent Systems 32(9), 926-946 (2017). https://doi.org/10.1002/int.21877

9. Roldán López de Hierro, A.F., Sesma-Sara, M., Špirková, J., Lafuente, J., Pradera, A., Mesiar, R., Bustince, H.: Curve-based monotonicity: a generalization of directional monotonicity. International Journal of General Systems (2019). https://doi.org/10.1080/03081079.2019.1586684

10. Lucca, G., Sanz, J., Dimuro, G., Bedregal, B., Asiain, M.J., Elkano, M., Bustince, H.: CC-integrals: Choquet-like copula-based aggregation functions and its application in fuzzy rule-based classification systems. Knowledge-Based Systems 119, 32-43 (2017). https://doi.org/10.1016/j.knosys.2016.12.004

11. Lucca, G., Sanz, J.A., Dimuro, G.P., Bedregal, B., Mesiar, R., Kolesárová, A., Bustince, H.: Preaggregation functions: Construction and an application. IEEE Transactions on Fuzzy Systems 24(2), 260-272 (2016). https://doi.org/10.1109/TFUZZ.2015.2453020

12. Lucca, G., Sanz, J.A., Dimuro, G.P., Bedregal, B., Bustince, H., Mesiar, R.: CFintegrals: A new family of pre-aggregation functions with application to fuzzy rulebased classification systems. Information Sciences 435, 94-110 (2018)

13. Mesiar, R., Kolesárová, A., Stupňanová, A.: Quo vadis aggregation? International Journal of General Systems 47(2), 97-117 (2018). https://doi.org/10.1080/03081079.2017.1402893

14. Paternain, D., Fernandez, J., Bustince, H., Mesiar, R., Beliakov, G.: Construction of image reduction operators using averaging aggregation functions. Fuzzy Sets and Systems 261, 87-111 (2015). https://doi.org/10.1016/j.fss.2014.03.008

15. Rousseeuw, P.J., Leroy, A.M.: Robust regression and outlier detection, vol. 589. John Wiley \& Sons (2005)

16. Sesma-Sara, M., Lafuente, J., Roldán, A., Mesiar, R., Bustince, H.: Strengthened ordered directionally monotone functions. Links between the different notions of monotonicity. Fuzzy Sets and Systems (In Press). https://doi.org/10.1016/j.fss.2018.07.007 
17. Sesma-Sara, M., Bustince, H., Barrenechea, E., Lafuente, J., Kolesárová, A., Mesiar, R.: Edge detection based on ordered directionally monotone functions. In: Advances in Fuzzy Logic and Technology 2017, pp. 301-307. Springer (2017)

18. Sesma-Sara, M., Mesiar, R., Bustince, H.: Weak and directional monotonicity of functions on Riesz spaces to fuse uncertain data. Fuzzy Sets and Systems (In press). https://doi.org/10.1016/j.fss.2019.01.019

19. Wilkin, T., Beliakov, G.: Weakly monotonic averaging functions. International Journal of Intelligent Systems 30(2), 144-169 (2015). https://doi.org/10.1002/int.21692 